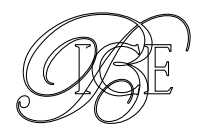

Raquel Rodríguez Méndez*

Ismael Bahillo Santoyo*

\section{DIFERENCIACIÓN DE MARCA E IMAGEN EN LA DISTRIBUCIÓN Y COMERCIALIZACIÓN DE ELECTRICIDAD Y GAS}

Este artículo analiza el contenido de la decisión jurídicamente vinculante, aprobada por la CNMC en septiembre de 2018, que establece obligaciones a varias empresas de distribución, comercialización de referencia de electricidad y comercialización de último recurso de gas en materia de separación de actividades, de acuerdo con lo establecido en las directivas del tercer paquete legislativo. Adicionalmente, esta decisión facilita al consumidor la diferenciación entre distribuidor y comercializador, y entre suministro a precio regulado y libre. Para ello se requiere que algunas de estas sociedades reguladas realicen cambios en la información, presentación de marca e imagen de marca, para que sean claramente distinguibles de la filial del mismo grupo que comercializa a precio libre, de forma consistente con el criterio seguido en otros países de nuestro entorno. De esta forma se elimina la ventaja que esta confusión otorga a ciertos agentes verticalmente integrados en favor de una mayor apertura y competencia en el mercado.

Palabras clave: separación de actividades, comercializador de referencia, comercializador de último recurso, competencia.

Clasificación JEL: D42, L43, L94, L95.

\section{Introducción}

Los procesos de liberalización en el sector energético que se han llevado a cabo desde principios del decenio de 1990 tuvieron como punto de partida la separación, dentro de la cadena de valor de cada sector, entre las actividades que se pueden desarrollar en competencia (generación eléctrica, producción o

\footnotetext{
* Dirección de Energía, CNMC.

Versión de enero de 2020.

DOI: https://doi.org/10.32796/bice.2020.3120.6965
}

importación de gas, comercialización) y aquellas otras que continúan reguladas como monopolios. En aquellas actividades que se pueden desarrollar en competencia, el papel del regulador es fundamental para promover una competencia efectiva, siendo necesaria la eliminación de las barreras de entrada y la consecución de un mercado único de la energía.

En septiembre de 2018, la Comisión Nacional de los Mercados y la Competencia (CNMC) aprobó una decisión jurídicamente vinculante dirigida a las empresas distribuidoras de electricidad (las de más de cien mil clientes) y $\triangleright$ 
gas, comercializadores de referencia y/o de último recurso. En particular, se requería a algunas de estas sociedades, en cumplimiento de la normativa en materia de separación de actividades, a realizar cambios en la información, presentación de marca e imagen de marca, para que fueran claramente distinguibles de la filial del mismo grupo empresarial que comercializa a precio libre, de manera que no puedan aprovecharse de su integración vertical para falsear la competencia. De esta forma, se espera también conseguir que los consumidores puedan diferenciar entre distribuidor y comercializador $y$, dentro de estos, entre los que suministran electricidad y/o gas a un precio regulado de los que lo suministran a un precio libremente pactado entre las partes, favoreciendo de esta manera la competencia a nivel minorista.

El objetivo de este artículo es dar a conocer las medidas establecidas por la CNMC en dicha resolución y los cambios en la denominación social e imagen de marca de distribuidores y comercializadores derivados de la misma que han tenido lugar recientemente, al objeto de que el consumidor se familiarice con los mismos y pueda diferenciar con claridad entre su distribuidor y su comercializador, regulado o libre, tanto en el sector eléctrico como en el sector gasista.

\section{Las directivas del tercer paquete}

Con la finalidad de armonizar y liberalizar el mercado interior de la energía de la Unión Europea se han adoptado desde 1996 medidas destinadas a alcanzar un mercado más competitivo, flexible y no discriminatorio, centrado en los clientes y en su protección. En general, este proceso se ha caracterizado por la necesidad de reforzar y ampliar los derechos de los consumidores.

El conjunto legislativo comunitario conocido como tercer paquete (Directiva 2009/72/CE ${ }^{1}$ y Directiva 2009/73/CE²) se promulgó con el objetivo de mejorar el funcionamiento del mercado interior de la energía, incorporando medidas destinadas a una mayor liberalización de los mercados de electricidad y gas, incluyendo disposiciones con el fin de alcanzar una mayor transparencia en los mercados minoristas en beneficio de los consumidores.

En estas directivas se reconoce, expresamente, que los intereses de los consumidores deben constituir el núcleo de las mismas, siendo preciso reforzar y garantizar sus derechos. Asimismo, se señala la necesidad de promover una competencia leal y el acceso sencillo a los diferentes suministradores, lo que permitirá a los consumidores disfrutar plenamente de las oportunidades de un mercado liberalizado de gas y electricidad.

Entre las medidas que el tercer paquete incorpora a la normativa comunitaria se encuentran las disposiciones que afectan a la presentación e imagen de marca de las distribuidoras que forman parte de grupos verticalmente integrados (desarrollan en el mercado, simultáneamente, actividades reguladas y libres) y que son el origen de los cambios que recientemente han afectado a estos elementos. Concretamente, los respectivos artículos 26.3 de las citadas directivas establecen que cuando el $\triangleright$

\footnotetext{
1 Directiva 2009/72/CE del Parlamento Europeo y del Consejo, de 13 de julio de 2009, sobre normas comunes para el mercado interior de la electricidad y por la que se deroga la Directiva 2003/54/CE. Recientemente se ha aprobado la Directiva 2019/944 del Parlamento Europeo y del Consejo, de 5 de junio de 2019, sobre normas comunes para el mercado interior de la electricidad y por la que se modifica la Directiva 2012/27/UE, mediante la cual queda derogada la Directiva 2009/72/CE con efectos a partir del 1 de enero de 2021.

2 Directiva 2009/73/CE del Parlamento Europeo y del Consejo, de 13 de julio de 2009, sobre normas comunes para el mercado interior del gas natural y por la que se deroga la Directiva 2003/55/CE.
} 
distribuidor forme parte de una empresa integrada verticalmente, los Estados miembros deben garantizar el control de sus actividades (a través de las autoridades reguladoras $u$ otros organismos competentes), de manera que no pueda aprovecharse de su integración vertical para falsear la competencia. En particular, indica que estos distribuidores no crearán confusión, en su información y en la presentación de la marca, respecto a la identidad de la sociedad de su mismo grupo empresarial que desarrolle la actividad de suministro. Así, esta medida pretende la eliminación de la ventaja que esta confusión otorga a ciertos agentes en favor de una mayor apertura y competencia en el mercado.

Estas disposiciones se incorporaron a la legislación nacional mediante el Real Decreto Ley $13 / 2012$, de 30 de marzo, que modificó el artículo 14 de la, entonces en vigor, Ley 54/1997, de 27 de noviembre -ahora artículo 12 de la Ley 24/2013, de 26 de diciembre, del Sector Eléctrico (LSE)-, y el artículo 63 de la Ley 34/1998, de 7 de octubre, del Sector de Hidrocarburos (LSH), ambos referidos a la separación de actividades.

En el sector eléctrico, el artículo 12.3 de la LSE obliga a las distribuidoras y comercializadoras de referencia que formen parte de un grupo de sociedades que desarrolle actividades libres y reguladas, en los términos previstos en dicha ley, a no crear confusión en su información y en la presentación e imagen de marca respecto a la identidad propia de las filiales de su mismo grupo que realicen actividades de comercialización. Para el sector de gas natural, el artículo 63.6 de la LSH establece la misma obligación para las distribuidoras de los grupos verticalmente integrados. Se destaca que la normativa española extiende la obligación de no confusión que la Directiva 2009/72/CE sobre el mercado eléctrico dispone para las distribuidoras también a las comercializadoras de referencia ${ }^{3}$.

En relación con el papel del regulador en cuanto al cumplimiento de esta norma, la $\mathrm{LSH}^{4}$ permite a la CNMC la adopción de medidas para promover el funcionamiento competitivo del sector energético y alcanzar ciertos objetivos, entre ellos: garantizar la efectiva disponibilidad y prestación de los servicios de suministro competitivos y de calidad en beneficio del mercado y de los consumidores, la protección de los consumidores de energía - en especial, los clientes vulnerables- y la compatibilidad de los procesos de intercambio de datos necesarios para que los clientes cambien de comercializador. Estos objetivos están integrados en diversas funciones que la Ley $3 / 2013$, de 4 de junio de creación de la CNMC, asigna al regulador, entre otras, la supervisión y control de los mercados, así como del grado y efectividad de su apertura y competencia, garantizar la transparencia y competencia de los mismos o la supervisión de la separación de actividades en el sector eléctrico y gasista.

En el ejercicio de las funciones que la normativa le atribuye, la CNMC inició en 2018 un procedimiento para dar cumplimiento efectivo a las obligaciones establecidas en los citados artículos 12.3 LSE y 63.6 LSH por su efecto directo en la elección de comercializador por parte del consumidor.

\footnotetext{
3 Tal y como queda expuesto en el apartado tercero de este artículo, sobre el mercado minorista, actualmente, en el segmento de suministro regulado en el sector gasista operan cuatro comercializadoras de último recurso (CUR), las cuales también comercializan electricidad a precio regulado en el mercado eléctrico bajo su condición de comercializadoras de referencia (COR).

4 Apartado sexto de la disposición adicional undécima de la LSH declarado expresamente en vigor por la disposición derogatoria b de la Ley $3 / 2013$, de 4 de junio, de creación de la Comisión Nacional de los Mercados y la Competencia.
} 


\section{El mercado minorista eléctrico y gasista en España: principales actores}

Dentro de los distintos agentes que intervienen en el suministro eléctrico y gasista a los consumidores, es importante diferenciar entre quién es el distribuidor, quién es el comercializador de referencia y/o de último recurso, y quién es el comercializador libre:

- La distribución es una actividad regulada que desarrolla el distribuidor y que tiene por objeto la trasmisión de la energía eléctrica desde las redes de transporte o directamente desde aquellos puntos en los que se genera la electricidad hasta los puntos en los que va al consumidor (en el caso del gas, el distribuidor lleva el gas desde la red de transporte hasta los puntos de consumo). A cambio de realizar dicha actividad, el distribuidor recibe una retribución regulada que paga el consumidor a través del correspondiente peaje. Desde el punto de vista del consumidor, es importante señalar que el distribuidor es el propietario de las líneas eléctricas que llevan la energía eléctrica a su vivienda, por tanto, el consumidor no puede cambiar de distribuidor. Adicionalmente, lo que paga el consumidor por ese servicio es un precio regulado similar con independencia de quién sea su comercializador.

En el sector eléctrico español hay más de trescientos distribuidores, pero destacan principalmente los distribuidores de cinco grandes grupos empresariales: i-DE Redes Eléctricas Inteligentes, SA (Grupo Iberdrola), EDISTRIBUCIÓN Redes Digitales, SLU (Grupo Endesa),
UFD Distribución Electricidad, SA (Grupo Naturgy), Viesgo Distribución Eléctrica, SL (Grupo Viesgo) y E-REDES Distribución Eléctrica (Grupo EDP). Por número de consumidores conectados a sus líneas, se sitúa en primer lugar EDISTRIBUCIÓN Redes Digitales, con el $41 \%$ de los puntos de suministro; seguido por i-DE Redes Eléctricas Inteligentes, con el 37\%; UFD Distribución Electricidad, con el 13\%; E-REDES Distribución Eléctrica, 2,3\%, y Viesgo Distribución Eléctrica, 1,8\%. Estos cinco grandes distribuidores tienen conectados a sus líneas el $95 \%$ del total de puntos de suministro eléctrico en España (29,4 miIlones a finales de 2018). En el sector gasista hay 19 distribuidores organizados en distintos grupos empresariales. A finales de 2018, el principal grupo distribuidor por puntos de suministro es Nedgia $^{5}$, con 5,95 millones de puntos de suministro (68\%); seguido de Nortegás ${ }^{6}$, con 0,95 millones (12\%); Madrileña Red de Gas, con 0,88 millones (11\%); Redexis $^{7}$, con 0,59 millones (7,5\%); y Gas Extremadura, con 75.358 puntos de suministro (1\%). Domus Mil Natural es una distribuidora de reciente creación que distribuye gas en Castilla-La Mancha, con 123 puntos de suministro a final de 2018.

- Por lo que se refiere al suministro, el comercializador es la empresa encargada de hacer llegar la electricidad y/o el $\triangleright$

5 Nedgia pertenece al Grupo Naturgy (anteriormente Gas Natural Fenosa) e incluye las siguientes distribuidoras: Nedgia Catalunya, Nedgia Madrid, Nedgia Cegas, Nedgia Castilla y León, Nedgia Andalucía, Nedgia Galicia, Nedgia Castilla-La Mancha, Nedgia Rioja, Nedgia Aragón, y Nedgia Redes de Distribución.

6 El Grupo Nortegás incluye Nortegás Energía, NED España Distribución y Gas Tolosa.

7 El Grupo Redexis incluye Redexis Gas y Redexis Gas Murcia. 
gas a los consumidores tras haberla comprado en el mercado mayorista. El consumidor tiene la posibilidad de elegir entre el suministro regulado y el suministro a un precio libremente pactado, siendo distintas las sociedades que suministran cada alternativa:

En el sector eléctrico, el suministro regulado lo realiza el Comercializador de Referencia (COR) a un precio denominado Precio Voluntario al Pequeño Consumidor (PVPC). Actualmente hay ocho COR, cuya denominación social y puntos de suministro a 31 de diciembre de 2018 se recogen en el Cuadro 1.

Por otra parte, a 31 de diciembre de 2018, había 315 comercializadoras suministrando a un precio libremente pactado entre las partes. Por número de puntos de suministro (Cuadro 2), destacan Iberdrola Clientes (36,6\% del mercado por número de puntos de suministro), Endesa Energía (29,6\%), Naturgy Iberia (12,3\%), EDP Energía y EDP Comercializadora $(5,1 \%)$ y Repsol Comercializadora de Electricidad y Gas (2,6\%). En conjunto, las comercializadoras $\triangleright$

CUADRO 1

COMERCIALIZADOR DE REFERENCIA

\begin{tabular}{|l|r|c|}
\hline Denominación social & $\begin{array}{c}\text { Puntos de } \\
\text { suministro }\end{array}$ & Porcentaje \\
\hline Energía XXI Comercializadora de Referencia, SLU & 5.076 .983 & 45,08 \\
Curenergía Comercializador de Último Recurso, SAU & 3.488 .285 & 30,98 \\
Comercializadora Regulada, Gas \& Power, SA & 2.233 .122 & 19,83 \\
Baser Comercializadora de Referencia, SA & 220.750 & 1,96 \\
Régsiti Comercializadora Regulada, SLU & 214.726 & 1,91 \\
Comercializador de Referencia Energético, SLU & 25.565 & 0,23 \\
Energía Ceuta XXI Comercializadora de Referencia, SAU & 1.817 & 0,02 \\
Teramelcor, SL & 43 & 0,00 \\
\hline Total & $\mathbf{1 1 . 2 6 1 . 2 9 1}$ & $\mathbf{1 0 0 , 0 0}$ \\
\hline Fuente: CNMC. & & \\
\hline
\end{tabular}

CUADRO 2

COMERCIALIZADOR POR N. ${ }^{\circ}$ DE PUNTOS DE SUMINISTRO

\begin{tabular}{|c|c|c|}
\hline Denominación social & $\begin{array}{l}\text { Puntos de } \\
\text { suministro }\end{array}$ & Porcentaje \\
\hline Iberdrola Clientes, SAU & 6.638 .033 & 36,57 \\
\hline Endesa Energía, SAU & 5.375 .220 & 29,62 \\
\hline Naturgy Iberia, SA & 2.226 .605 & 12,27 \\
\hline EDP Energía, SAU y EDP Comercializadora, SA & 932.279 & 5,14 \\
\hline Repsol Comercializadora de Electricidad y Gas, SLU & 475.087 & 2,62 \\
\hline CIDE HC Energía, SA & 406.706 & 2,24 \\
\hline Fenie Energía, SA & 360.007 & 1,98 \\
\hline Clidom Energy, SA & 143.305 & 0,79 \\
\hline Otros & 1.592 .114 & 8,77 \\
\hline Total & 18.149.356 & 100,00 \\
\hline
\end{tabular}


de los principales grupos energéticos tradicionales suministraban el $86,5 \%$ del total de puntos suministrados en el mercado a precio libre ${ }^{8}$. El resto de comercializadoras, las llamadas independientes, suministraban electricidad al $13,5 \%$ de los consumidores, destacando principalmente CIDE HC Energía $(2,2 \%)$, Fenie Energía (2,0\%) y Clidom Energy (0,8\%).

En el sector gasista, el suministro regulado lo realizan los comercializadores de último recurso (CUR) a una tarifa de último recurso (TUR). Actualmente hay cuatro CUR, cuya denominación social y

8 Se incluye a Repsol Electricidad y Gas dentro de este grupo al pertenecer durante la mayor parte de 2018 al Grupo VIESGO. puntos de suministro a 31 de diciembre de 2018 se recogen en el Cuadro 3.

Por su parte, a 31 de diciembre de 2018 , 74 compañías han suministrado gas natural a clientes finales a un precio libremente pactado entre las partes. Por número de puntos de suministro, destacan Naturgy Iberia (45,8\% del mercado por número de puntos de suministro), Endesa Energía $(20,7 \%)$, Iberdrola Clientes (16,0\%), EDP España (13,4\%) y Repsol Comercializadora de Electricidad y Gas $(1,6 \%)$. Por tanto, las comercializadoras de los principales grupos energéticos tradicionales suministraban el $97,7 \%$ del total de los puntos de suministro del mercado libre. El resto de comercializadoras, las llamadas independientes, $D$

CUADRO 3

COMERCIALIZADOR DE ÚLTIMO RECURSO

\begin{tabular}{|c|c|c|}
\hline Denominación social & $\begin{array}{l}\text { Puntos de } \\
\text { suministro }\end{array}$ & Porcentaje \\
\hline Comercializadora Regulada, Gas \& Power, SA & 1.290 .001 & 81,13 \\
\hline Energía XXI Comercializadora de Referencia, SLU & 232.669 & 14,63 \\
\hline Baser Comercializadora de Referencia, SA & 51.376 & 3,23 \\
\hline Curenergía Comercializador de Último Recurso, SAU & 15.972 & 1,00 \\
\hline Total & 1.590 .018 & 100,00 \\
\hline
\end{tabular}

CUADRO 4

COMERCIALIZADOR POR N. ${ }^{\circ}$ DE PUNTOS DE SUMINISTRO

\begin{tabular}{|c|c|c|}
\hline Denominación social & $\begin{array}{l}\text { Puntos de } \\
\text { suministro }\end{array}$ & Porcentaje \\
\hline Naturgy Iberia, SA & 2.886 .977 & 36,59 \\
\hline Endesa Energía, SAU & 1.302 .044 & 16,50 \\
\hline Iberdrola Clientes, SAU & 1.008 .535 & 12,78 \\
\hline EDP España, SA & 843.716 & 10,69 \\
\hline Repsol Comercializadora de Electricidad y Gas, SLU & 101.639 & 1,29 \\
\hline Fenie Energía, SA & 22.553 & 0,29 \\
\hline Aldro Energía y Soluciones, SLU & 17.490 & 0,22 \\
\hline Otros & 117.153 & 1,48 \\
\hline Total & 2.886.977 & 36,59 \\
\hline
\end{tabular}


suministraban electricidad al $2,3 \%$ de los consumidores, destacando Fenie Energía $(0,4 \%)$ y Aldro Energía y Soluciones $(0,3 \%)$.

Es importante destacar que hay varios grupos integrados que están presentes tanto en distribución como en comercialización regulada y libre. Es el caso del grupo Iberdrola (i-DE Redes Eléctricas Inteligentes, Curenergía Comercializador de Último Recurso e Iberdrola Clientes), el grupo Endesa (EDISTRIBUCIÓN Redes Digitales, Energía XXI Comercializadora de Referencia y Endesa Energía $\left.{ }^{9}\right)$, el grupo Naturgy (UFD Distribución Electricidad y Nedgia, Comercializadora Regulada, Gas \& Power y Naturgy Iberia) o el grupo EDP (E-REDES Distribución Eléctrica, Baser Comercializadora de Referencia, EDP Energía y EDP Comercializadora). También era el caso del grupo Viesgo hasta que sus comercializadoras fueron adquiridas por el grupo Repsol el 2 de noviembre de 2018.

En relación con los distintos agentes que participan en el suministro energético a nivel minorista, es importante señalar que diversos indicadores del Panel de Hogares que realiza la CNMC evidencian las dificultades de un número significativo de consumidores para comprender información básica del mercado: apenas 1 de cada 5 consumidores encuestados en el Panel de Hogares del segundo trimestre de 2019 declara conocer la diferencia entre el suministro regulado y el precio libremente pactado (22\% en electricidad y $20 \%$ en gas). De forma similar, un $64 \%$ de consumidores de electricidad y un $70 \%$ de gas desconocen en cuál de estos mercados contrataron su suministro. De igual forma, un

9 Asimismo, el 24 de julio de 2018, el Grupo Endesa cerró la compra de todo el capital social de la Empresa de Alumbrado Eléctrico de Ceuta. promedio del $38 \%$ de los hogares encuestados apunta como motivo de insatisfacción la falta de información sobre las condiciones de suministro y contractuales, según las sucesivas encuestas semestrales del Panel de Hogares durante el periodo 2015-2018. Por tanto, es razonable suponer que, si solo 1 de cada 5 consumidores conoce la diferencia entre suministro regulado y a precio libre, el porcentaje de consumidores que es capaz de distinguir entre comercializadoras en mercado regulado, mercado libre y distribuidores es aún inferior. En ese sentido, la similitud en la denominación social y en la imagen de marca empleada por distribuidores y comercializadores del mismo grupo empresarial no favorecía que el consumidor pudiese distinguir entre cada una de estas sociedades.

Por otra parte, se observa una fuerte fidelización entre el distribuidor y las comercializadoras de su mismo grupo: por ejemplo, durante el periodo 2013-2018 se han producido 3.182.266 altas de puntos de suministro eléctrico en el área de distribución de los cinco principales distribuidores, siendo 2.814.766 (el $88,5 \%$ del total) los puntos suministrados por comercializadoras pertenecientes al mismo grupo empresarial de dichos distribuidores. Respecto al sector gasista, para el conjunto del periodo 2013-2018 se han producido 885.878 altas de puntos de suministro en el área de distribución del Grupo Naturgy, siendo suministrados el $80,1 \%$ de las nuevas altas $(709.758$ puntos) por las comercializadoras pertenecientes a dicho grupo.

Asimismo, por lo que se refiere a los cambios de comercializador que se producen desde el comercializador de referencia hacia el comercializador libre del mismo grupo empresarial, es destacable para el sector eléctrico que de los casi 7,7 millones de consumidores que han salido del COR durante el periodo $D$ 
2013-2018, el 76\% de ellos (5,8 millones) cambió a la comercializadora libre perteneciente al mismo grupo empresarial. Por su parte, en el periodo analizado (2013-2018), 657.247 consumidores han cambiado de suministrador de gas natural desde el comercializador de último recurso, y más del $52 \%$ de ellos ha elegido a las comercializadoras libres del mismo grupo empresarial para su suministro.

\section{Medidas incorporadas en la Decisión Jurídicamente Vinculante de la CNMC (DJV)}

Además de las conclusiones que se derivan del Panel de Hogares mencionadas anteriormente, en diversas ocasiones la CNMC ha identificado la existencia de confusión en la información, presentación e imagen de marca en los grupos verticalmente integrados tanto en informes de supervisión ${ }^{10}$ en los que incorporaba recomendaciones de actuación al respecto, como en algunas de sus resoluciones ${ }^{11}$. También terceros han comunicado al regulador esta situación a través de denuncias y, por su parte, el Consejo de Reguladores Europeos de Energía (CEER), en un informe de $2016^{12}$ sobre el grado de cumplimiento de las obligaciones de separación de actividades de los distribuidores de gas y electricidad integrados verticalmente, identificó países en los que existía confusión en

\footnotetext{
10 Informe de supervisión de las ofertas del mercado minorista de gas y electricidad recogidas en el comparador de ofertas de la CNMC en junio de 2014, Informe de supervisión del mercado minorista de electricidad de la CNMC en 2015, así como los informes de supervisión de los servicios de atención al cliente de la CNMC de 2015 y 2018.

11 Resolución de 28 de julio de 2016 C-0758/16 Gas Natural Fenosa/ GLP Repsol Butano-Activos; Resolución de 28 de julio de 2016 C-0759/16 Naturgas/GLP Repsol Butano Activos; Resolución de 7 de diciembre de 2016 C-0812/16 Gas Natural Fenosa/GLP Cepsa-Activos y Resolución de 18 de febrero de la CNMC S/DC/O515/14 Comercializadoras de electricidad.

12 CEER (1 de abril de 2016). Status Review on the Implementation of Distribution System Operators' Unbundling Provisions of the $3^{\text {rd }}$ Energy Package.
}

la imagen de marca e identidad corporativa de los grupos verticalmente integrados, señalando a España entre aquellos con mayor número de empresas en tal situación.

Ante la continuidad de este estado de confusión, la CNMC inició un procedimiento que supuso la aprobación, en septiembre de 2018, de una decisión jurídicamente vinculante destinada al cumplimiento efectivo de las obligaciones recogidas en los artículos 12.3 LSE y 63.6 LSH y que venían a sumarse a las obligaciones ya existentes de separación de actividades jurídica y funcional para los distribuidores de grupos verticalmente integrados.

La DJV señala que cualquier barrera en el mercado (siendo una de ellas la confusión del consumidor por la marca e imagen de marca ${ }^{13}$ ) provoca una distorsión en la competencia efectiva que favorece a las comercializadoras libres que se identifican de forma equívoca con respecto a la imagen del distribuidor o del comercializador en el mercado regulado de su mismo grupo empresarial, teniendo como efecto el falseamiento de la competencia, concediendo una ventaja a los comercializadores de los grupos integrados. Por tanto, la finalidad del procedimiento es la protección al consumidor, especialmente doméstico, en su decisión de elegir comercializador (derecho recogido en el artículo 44.1.c de la LSE y en el artículo 57.bis.b de la LSH), pretendiendo que aquel no vea alterada su libre elección de suministrador por el hecho de que los distribuidores y comercializadores en el mercado regulado de los grupos integrados creen confusión en su identidad con la del comercializador del grupo que opera en el mercado libre.

Así, la DJV exige a las empresas distribuidoras y comercializadoras en mercado $D$

13 CEER Benchmarking report on removing barriers to entry for energy suppliers in EU retail energy markets, de 1 de abril de 2016. 
regulado ${ }^{14}$ de los grupos verticalmente integrados que adopten las siguientes medidas:

- No crear confusión en su presentación de marca (denominación social) respecto a la identidad propia de las sociedades de su mismo grupo que realicen actividades de comercialización en el mercado libre.

- No crear confusión en su imagen de marca (logotipo) respecto a la identidad propia de las sociedades de su mismo grupo que realicen actividades de comercialización en el mercado libre.

- No crear confusión en la información que remitan o intercambien con el consumidor doméstico a través de cualquier canal de comunicación (telefónico, escrito, web, presencial) respecto de las sociedades del grupo que realicen actividades de comercialización en el mercado libre.

Asimismo, en la DJV se señalan los criterios para materializar las medidas anteriores. Si bien la denominación social de la sociedad distribuidora o comercializadora en el mercado regulado debe permitir al consumidor identificar correctamente la actividad que desarrolla, se considera que este elemento no es suficiente para eliminar el riesgo de confusión si, además, la denominación incluye el nombre del grupo de sociedades y la referencia al grupo también se incorpora en la denominación social de la comercializadora libre. En cuanto al logotipo de estas sociedades, se advierte que el uso de palabras, letras, cifras, figuras,

\footnotetext{
14 Distribuidoras de gas y electricidad (para este sector concreto, aquellas con más de cien mil clientes conectados a sus redes) y comercializadoras de referencia o de último recurso que formen parte de grupos empresariales que integren, igualmente, sociedades que comercializan electricidad y gas natural en el mercado libre.
}

signos, dibujos, elementos o símbolos comunes o idénticos a los empleados para el logotipo de la empresa comercializadora del grupo en el mercado libre pueden crear confusión en el consumidor. No obstante, se permite la indicación de la pertenencia al grupo, pero con un tamaño menor y con una disposición inferior y menos visible, con respecto a los elementos que conforman la imagen de la sociedad.

La situación de partida, en cuanto a las denominaciones sociales de las sociedades afectadas por la DJV, se caracterizaba, en general, por la incorporación de la mención a la actividad que desarrolla la sociedad (distribución, comercialización de último recurso, comercializadora de último recurso o comercializador de referencia) como elemento diferenciador, así como la inclusión de la identificación al grupo de sociedades que también aparece en la denominación social de la comercializadora en el mercado libre. Respecto a sus logotipos, en general, mostraban elementos comunes (símbolos, palabras y colores) respecto de los logotipos de las comercializadoras libres del grupo.

Tras el análisis realizado caso por caso, la DJV identificó los aspectos que generaban confusión en el consumidor y que, al menos, habían de ser modificados por cada agente. No obstante, también concluyó que algunas sociedades no generaban confusión en su presentación e imagen de marca. Así, respecto de la denominación social, no inducían a confusión la distribuidora eléctrica del grupo EDP, las sociedades del Grupo Naturgy (distribuidoras de gas y electricidad, así como la comercializadora en el mercado regulado ${ }^{15}$ ) y la comercializadora $\triangleright$

15 La comercializadora en el mercado regulado del Grupo Naturgy modificó su presentación e imagen de marca en 2018 a lo largo de la tramitación de la DJV. A principios de 2018 y con anterioridad al inicio del procedimiento DJV, Gas Natural Fenosa lanzó Nedgia como la nueva marca para el negocio de distribución de gas, modificando las denominaciones sociales de las distribuidoras gasistas incorporando este término. 
en el mercado regulado del grupo Gaselec. Con respecto a la presentación de marca, no inducían a confusión los logotipos de las sociedades del Grupo Naturgy (distribuidoras y suministradora en el mercado regulado) y el asociado a la comercializadora en el mercado regulado del grupo Gaselec.

En cuanto al grupo Viesgo, tras la venta de su negocio de suministro de gas y electricidad a Repsol en 2018, la distribuidora Viesgo Distribución Eléctrica dejó de ser sujeto obligado al cumplimiento de la DJV.

La DJV estableció un plazo de seis meses para la implementación de medidas, permitiendo que los sujetos obligados remitieran sus propuestas a la CNMC para su valoración ${ }^{16}$.

Actualmente, las sociedades afectadas ya han procedido a modificar sus denominaciones sociales y logotipos, mostrándose en los siguientes cuadros los cambios que se han llevado a cabo. El Cuadro 5 compara la actual denominación social y el logotipo de las distribuidoras de electricidad con respecto a la situación previa a la DJV y el Cuadro 6 muestra el mismo ejercicio, pero, en este

16 Nota de prensa CNMC: $h$ ttps://www.cnmc.es/node/375417 caso, en relación con las comercializadoras de energía eléctrica y gas natural en el mercado regulado.

Tal y como puede comprobarse, las nuevas denominaciones sociales de las sociedades no incluyen referencia al grupo al que pertenecen. También se aprecia que los nuevos logotipos son completamente distintos a los anteriores y, en general, incluyen en un menor tamaño la mención al grupo del que forma parte la empresa, lo que permite al consumidor disponer de esta información de forma inmediata.

El logotipo es el signo gráfico que identifica visualmente a la sociedad, pero también la denominación social la identifica, y ambos elementos son distintivos, igualmente, de los productos o servicios de la sociedad. Así, es importante destacar que, en la medida en que ambos elementos trasladen información clara y sin ambigüedad, se facilitará que el consumidor sea consciente de su elección comercial y se favorecerá y simplificará la comparación de ofertas y mercados, eliminando o minimizando obstáculos para que el consumidor pueda ejercer su derecho a elegir libremente comercializador de forma inequívoca y sin confusión.

CUADRO 5

MODIFICACIÓN DE LA DENOMINACIÓN SOCIAL E IMAGEN DE MARCA DE LAS DISTRIBUIDORAS ELÉCTRICAS

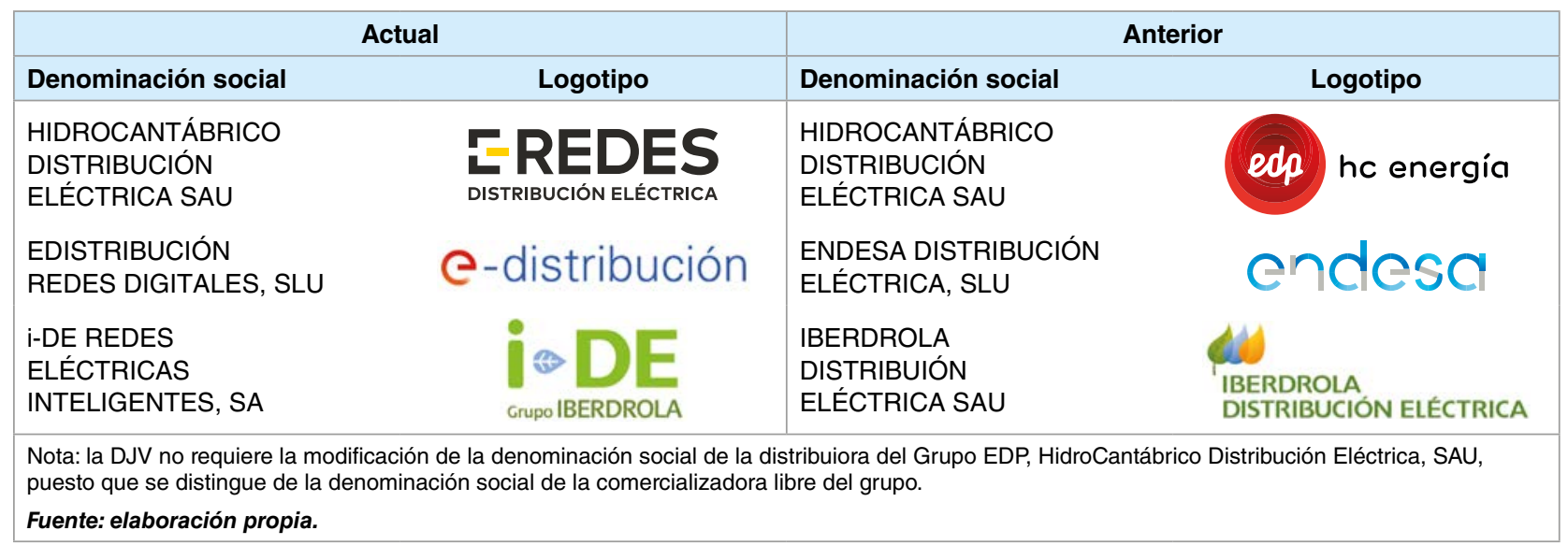


CUADRO 6

MODIFICACIÓN DE LA DENOMINACIÓN SOCIAL E IMAGEN DE MARCA DE LAS COMERCIALIZADORAS REGULADAS

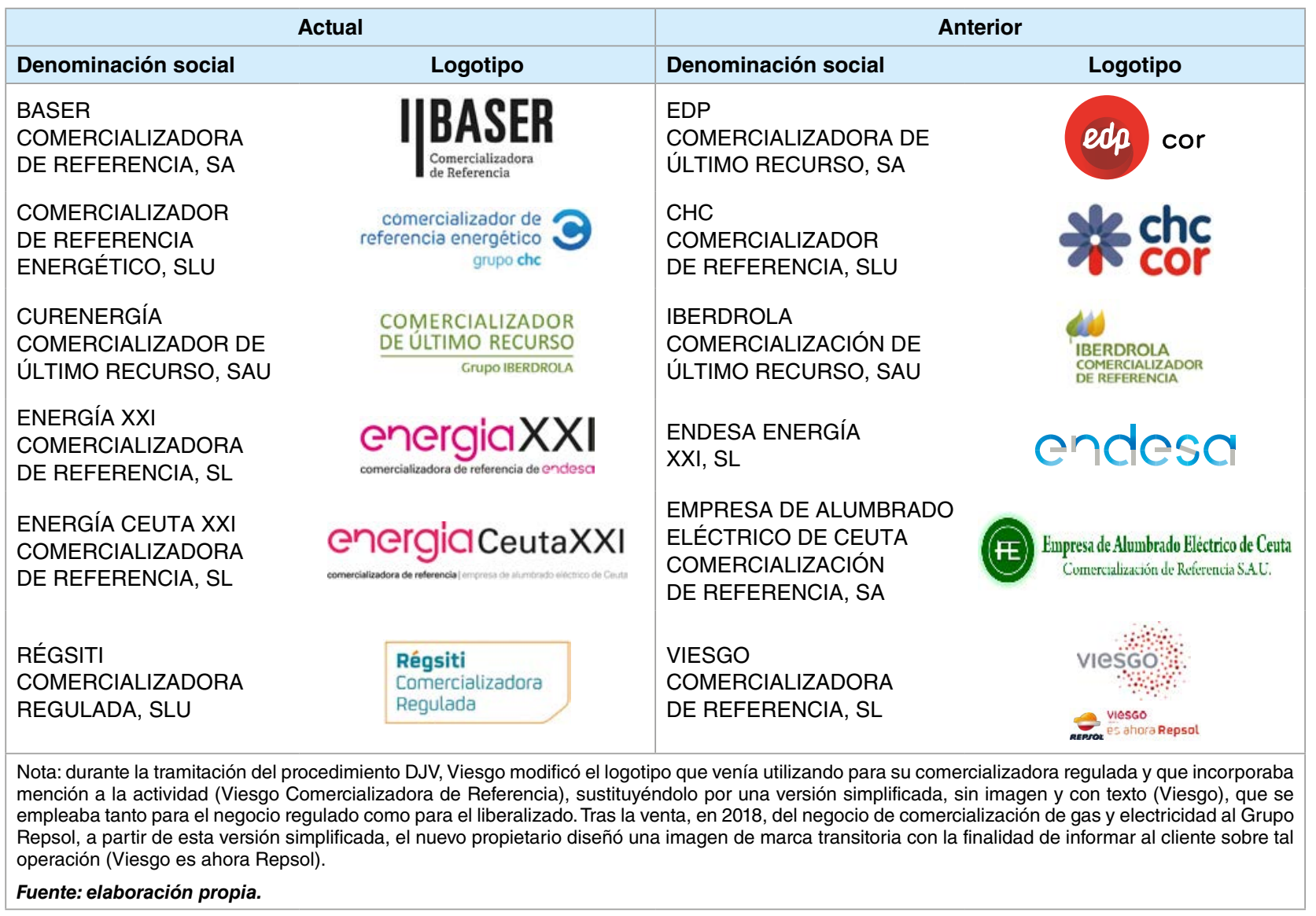

Finalmente, el Cuadro 7 incorpora la actual denominación social y logotipo, tanto de la comercializadora en el mercado libre como de la comercializadora en el mercado regulado del mismo grupo, reflejando las sustanciales diferencias entre ambas categorías.

Procede señalar que la DJV exige que las distribuidoras y comercializadoras en el mercado regulado de los grupos verticalmente integrados se identifiquen claramente y sin confusión en todas las comunicaciones e informaciones que intercambie con el cliente a través de cualquier canal (facturas, e-mail, teléfono, web, área cliente, app, oficinas de atención presencial), de manera que las nuevas denominaciones sociales y logotipos se trasladan e incluyen en todas las herramientas de comunicación con el consumidor.

\section{Experiencia en materia de marca en países de nuestro entorno}

Como se ha señalado, la obligación del distribuidor de gas natural y de electricidad a no crear confusión en su información y en la presentación de marca e imagen de marca, respecto a la identidad de la filial comercializadora perteneciente al grupo integrado verticalmente, procede de la normativa europea (artículos 26.3 de la Directiva 2009/72/CE y de la Directiva 2009/73/CE, respectivamente), por consiguiente, son varios los países de la Unión Europea en los que se han tomado o están tomando medidas similares a las aprobadas por la CNMC. En este sentido cabe destacar el reciente informe Implementation of TSO $\square$ 
Raquel Rodríguez Méndez e Ismael Bahillo Santoyo

CUADRO 7

COMPARACIÓN DE LA ACTUAL DENOMINACIÓN SOCIAL Y LOGOTIPO DE LAS COMERCIALIZADORAS REGULADAS Y LAS COMERCIALIZADORAS LIBRES

\begin{tabular}{|c|c|c|c|c|}
\hline \multicolumn{2}{|c|}{ Comercializadora regulada } & \multicolumn{3}{|c|}{ Comercializadora libre } \\
\hline $\begin{array}{l}\text { Denominación } \\
\text { social/nombre }\end{array}$ & Logotipo/marca & $\begin{array}{l}\text { Denominación } \\
\text { social/nombre }\end{array}$ & & Logotipo/marca \\
\hline $\begin{array}{l}\text { BASER } \\
\text { COMERCIALIZADORA } \\
\text { DE REFERENCIA, SA }\end{array}$ & 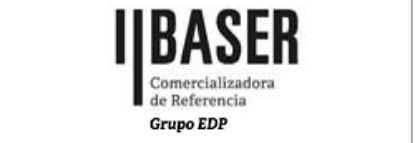 & EDP ENERGIA, SAU & & edp \\
\hline $\begin{array}{l}\text { COMERCIALIZADOR } \\
\text { DE REFERENCIA } \\
\text { ENERGÉTICO, SLU }\end{array}$ & $\underset{\substack{\text { comercializador de } \\
\text { referenciapo chc }}}{\mathbf{Q}}$ & CIDE HCENERGÍA, SA & & \\
\hline $\begin{array}{l}\text { CURENERGÍA } \\
\text { COMERCIALIZADOR DE } \\
\text { ÚLTIMO RECURSO, SAU }\end{array}$ & $\begin{array}{r}\text { COMERCIALIZADOR } \\
\text { DE ÚLTIMO RECURSO } \\
\text { Grupo IBERDROLA }\end{array}$ & $\begin{array}{l}\text { IBERDROLA } \\
\text { CLIENTES, SAU }\end{array}$ & & IBERDROLA \\
\hline $\begin{array}{l}\text { ENERGÍA XXI } \\
\text { COMERCIALIZADORA } \\
\text { DE REFERENCIA, SL }\end{array}$ & energia XXI & ENDESA ENERGÍA, SL & & \\
\hline $\begin{array}{l}\text { ENERGÍA CEUTA XXI } \\
\text { COMERCIALIZADORA } \\
\text { DE REFERENCIA, SL }\end{array}$ & ICeutaXXI & $\begin{array}{l}\text { EMPRESA DE } \\
\text { ALUMBRADO ELÉCTRICO } \\
\text { DE CEUTA, SA }\end{array}$ & FE & Empresa de Alumbrado Electrico de Ceuta \\
\hline $\begin{array}{l}\text { COMERCIALIZADORA } \\
\text { REGULADA, } \\
\text { GAS \& POWER, SA }\end{array}$ & $\begin{array}{r}\text { comercializadora } \\
\text { regulada } \\
\text { Grupo Naturgy }\end{array}$ & NATURGY IBERIA, SA & & \\
\hline $\begin{array}{l}\text { RÉGSITI } \\
\text { COMERCIALIZADORA } \\
\text { REGULADA, SLU }\end{array}$ & $\begin{array}{l}\text { Régsiti } \\
\text { Comercializadora } \\
\text { Regulada }\end{array}$ & $\begin{array}{l}\text { REPSOL } \\
\text { COMERCIALIZADORA } \\
\text { DE ELECTRICIDAD Y } \\
\text { GAS, SLU }\end{array}$ & & \\
\hline TERAMELCOR, SL & Teramelcor & $\begin{array}{l}\text { GASELEC } \\
\text { DIVERSIFICACIÓN, SL }\end{array}$ & & $\underset{\text { icación }}{\text { elec }}$ \\
\hline $\begin{array}{l}\text { Nota: la DJV analizó también a } \\
\text { la comercializadora libre (Gas } \\
\text { requirió actuación alguna resp }\end{array}$ & $\begin{array}{l}\text { upo Gaselec concluyendo en la ause } \\
\text { Diversificación, SL), tanto en relació } \\
\text { de la modificación de estos elemen }\end{array}$ & $\begin{array}{l}\text { encia de confusión entre la comer } \\
\text { on con la denominación social con } \\
\text { tos. }\end{array}$ & $\begin{array}{l}\text { adora c } \\
\text { la ima }\end{array}$ & $\begin{array}{l}\text { eferencia (TERAMELCOR, SL) } \\
\text { de marca, por lo tanto, no se I }\end{array}$ \\
\hline Fuente: CNMC (https://blog.C & (2) & ercializadoras-de-referencia-ca & 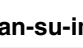 & \\
\hline
\end{tabular}

and DSO Unbundling Provisions ${ }^{17}$ del Consejo Europeo de Reguladores de la Energía (CEER), en el que se lleva a cabo un análisis del estado de la implementación de las disposiciones establecidas en las directivas del tercer paquete sobre separación de actividades en los operadores de redes de transporte (TSO) y operadores de redes de distribución (DSO) desde 2015 hasta mediados de 2018.

17 Implementation of TSO and DSO Unbundling Provisions. Update and Clean Energy Package Outlook. CEER Status Review. Ref: C18LAC-02-08, 14 de junio de 2019.
En lo que se refiere a la denominación social e imagen de marca, el informe destaca, en primer lugar, los desarrollos regulatorios que se han llevado a cabo en varios países (por ejemplo, Dinamarca, Italia, Grecia y Noruega) en materia de marca como parte de la regulación en materia de separación de actividades. Dentro de estos países destaca Italia, donde el regulador, ARERA, con el fin de evitar cualquier confusión con respecto a la identidad de la filial de comercialización de la empresa verticalmente integrada, determinó que tanto $\triangleright$ 
los distribuidores de electricidad como los de gas debían llevar a cabo un cambio de marca (Delibera 296/2015/R/com y desarrollos posteriores, en particular, Delibera 561/2018/E/EEL). En Gran Bretaña y Alemania, Ofgem y BNetzA han identificado un aumento en el número de DSO cuya identidad podría crear confusión con la filial de comercialización de la empresa verticalmente integrada. Ofgem y BNetzA requirieron a dichos DSO tomar las medidas correspondientes para evitar la confusión.

En Francia, como resultado de un análisis llevado a cabo por la CRE (ERDF), la principal distribuidora eléctrica perteneciente a EDF cambió su logo e identidad corporativa a ENEDIS. Adicionalmente, en 2018, la CRE ha requerido a otra distribuidora (Gérédis) que cambie su logo dado que podía generar confusión con el de la comercializadora de dicha empresa verticalmente integrada. En Luxemburgo, el grupo verticalmente integrado Enovos International SA, ha cambiado su denominación social en octubre de 2016, a Encevo $\mathrm{SA}$, al objeto de evitar cualquier posible confusión entre el holding y sus filiales Enovos Luxembourg SA y Creos Luxembourg SA.

En agosto de 2019, el regulador portugués, ERSE, anunció el cambio de denominación social e imagen de marca de la comercializadora en el mercado regulado de EDP Serviço Universal, que pasará a denominarse SU Electricidade para diferenciarse de la comercializadora en el mercado liberalizado EDP Comercial. Está todavía pendiente el cambio de denominación social e imagen de marca de EDP Distribuição, habiendo registrado la sociedad nombres como E-Redes (adoptado en España), Inodis y Edis.

El Cuadro 8 muestra algunos cambios recientes en los logos de las distribuidoras de grupos verticalmente integrados.
El informe de CEER concluye que en aquellos Estados miembros en los que se han llevado a cabo procesos de cambio de denominación y de imagen de marca la mayoría de los reguladores han considerado que estos se han completado de forma satisfactoria tanto en gas como en electricidad. Por otra parte, varios reguladores de distintos Estados miembros han detectado casos de incumplimiento, pero, aunque la legislación nacional de algunos países (por ejemplo, Dinamarca, Alemania, Francia, Gran Bretaña) permite la imposición de sanciones, hasta el momento ningún regulador ha tenido que llegar a imponer sanciones por este motivo.

\section{Conclusiones}

La Resolución de la CNMC de septiembre de 2018, que se analiza en este artículo, tiene como objetivo que se cumpla con los requisitos legalmente establecidos en materia de separación de actividades, de forma que se facilite la identificación de los distintos agentes dentro de empresas verticalmente integradas, favoreciendo de esta forma la competencia en el mercado minorista $y$, en última instancia, beneficiando al consumidor.

Es muy relevante que el consumidor distinga entre las actividades de distribución y suministro $y$, dentro de estas, entre suministro regulado y suministro a un precio libremente pactado. Solo de esta forma podrá sacar beneficio a su elegibilidad. Para ello, la resolución persigue una clara diferenciación entre las distintas sociedades dentro de empresas verticalmente integradas.

En 2016, la UE inició la tramitación de diversas normas presentadas en el Paquete de Invierno (o Paquete de Energía Limpia) como $\triangleright$ 
Raquel Rodríguez Méndez e Ismael Bahillo Santoyo

CUADRO 8

COMPARATIVA DE LOGOS DE LAS DISTRIBUIDORAS DE GRUPOS VERTICALMENTE INTEGRADOS

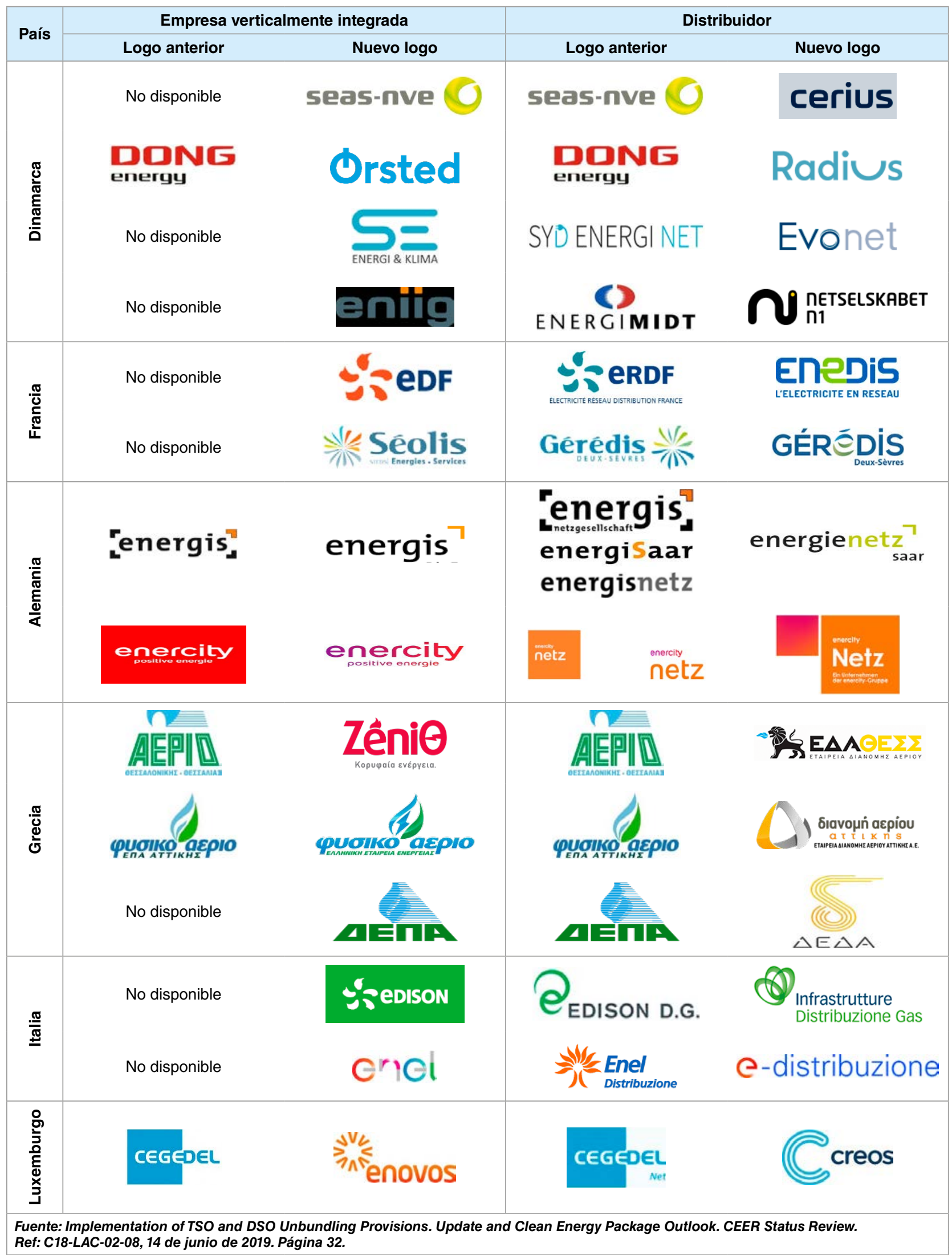


revisión del marco normativo de la política energética, habiéndose completado este proceso en 2019. Este conjunto de normas persigue, principalmente, tres objetivos: anteponer la eficiencia energética, lograr el liderazgo mundial en materia de energías renovables y ofrecer un trato justo a los consumidores. Asimismo, considera al consumidor como un «pilar fundamental de la Unión de la Energía» y propone reformar el mercado de la energía con la finalidad de capacitarlo y permitirle controlar mejor sus opciones energéticas, lo que supondrá para el consumidor disponer de una información de mayor calidad y la posibilidad de desempeñar un papel más activo y de un mayor control de sus costes energéticos. En este sentido cabe recordar que, tal y como establece el artículo 15 de la Directiva (UE) 2019/944 del Parlamento Europeo y del Consejo sobre normas comunes para el mercado interior de la electricidad, los Estados miembros deben garantizar que los clientes finales tengan derecho a actuar como clientes activos sin estar sujetos a requisitos técnicos o administrativos, procedimientos o gastos, desproporcionados o discriminatorios, ni a tarifas de acceso a la red que no reflejen los costes.

En este escenario que proyecta la figura de un consumidor más participativo y proactivo es fundamental que las Administraciones Públicas trabajen en fortalecer y ayudar al consumidor para poder afrontar los retos y oportunidades, presentes y futuros, que le ofrece el actual proceso de transición energética. En este sentido, una vez implementada la resolución de la CNMC, es relevante difundir los objetivos de la misma y los cambios que se han llevado a cabo para que el consumidor los conozca y se familiarice con los nuevos términos, de manera que la identificación clara y sin confusión de los distintos operadores y mercados en los que actúan le permita ejercer su derecho a elegir libremente suministrador de electricidad y gas con mayores garantías y sin injerencias. 